\title{
Enriquecimiento de señal para activación de frenos ABS en automóviles
}

\author{
José Vázquez Quezada ${ }^{1}$, Juan Villegas-Cortez ${ }^{1}$, Iván Vázquez-Álvarez ${ }^{1}$, Carlos \\ Avilés-Cruz ${ }^{1}$, Ismael Osuna-Galán ${ }^{2}$, Yolanda Pérez-Pimentel ${ }^{2}$ \\ 1 Universidad Autónoma Metropolitana, Azcapotzalco, Departamento de \\ Electrónica, México, D.F. \\ 2 Universidad Politécnica de Chiapas, \\ Tuxtla Gtz., Chiapas, México \\ jose.pp.vq@gmail.com, \{juanvc, iva, caviles\}@azc.uam.mx, \\ $\{$ iosuna, ypimentel\}@upchiapas.edu.mx
}

\begin{abstract}
Resumen. El sistema de frenos ABS es ampliamente usado en los automóviles de uso doméstico por su alta eficiencia y mayor aporte de seguridad, además con la masificación de su uso, el costo de éste se ha reducido. Actualmente la activación de este sistema de frenos depende en gran medida de la aplicación abrupta del pedal por parte del conductor del vehículo, por lo que el funcionamiento de éste depende principalmente de las aptitudes del conductor. En este documento se presenta una propuesta de mejoramiento del sistema de frenos ABS, considerando un enriquecimiento de la señal de activación, usando visión por computadora del espacio de rodamiento (camino transitado), tomando la señal proporcionada por el módulo de visión, a fin de tener activaciones más rápidas e independientes del conductor, y antes de que los sensores detecten que se está produciendo un bloqueo desde los neumáticos. Aquí se muestra el detalle del prototipo de mezclado-unión de las señales (computadora del automóvil + la incorporación de visión), así como la implementación simulada en el laboratorio con resultados prometedores.
\end{abstract}

Palabras clave: procesamiento digital de señales, FPGA, ABS.

\section{Introducción}

Actualmente el Sistema de Frenos Antibloqueo (Antilock Brake System, ABS), se utiliza en situaciones donde se debe detener un vehículo que viaja a una velocidad considerable sin perder maniobrabilidad del automóvil durante el proceso de frenado [1], e.g. en una carretera resbaladiza por condiciones climáticas, factores humanos o condiciones de la superficie misma. El sistema de frenos ABS permite que se mantenga el control del vehículo en el frenado sin perder el arrastre o tracción, evitando un derrape o amarre, incluso en pistas cubiertas de hielo.

El funcionamiento es simple, cada rueda tiene un sensor de velocidad angular (generalmente un sensor magnético), cuando la velocidad angular de una de 
las ruedas cambia muy rápido con respecto a las demás se detecta una posible condición de bloqueo, entonces las válvulas presurizan, o liberan presión, en dicho neumático según lo necesite para evitar el amarre y poder frenar y a su vez evadir obstáculos de manera controlada si es preciso mientras se detiene. Aunque hay varios modelos de ABS [2], [3] , todos constan de cuatro componentes básicos que se mencionan continuación y se muestran en la figura 1.

1. Sensores de velocidad: Cada rueda tiene un sensor que estima la velocidad angular de cada rueda, con lo cual es posible determina cuando alguna rueda está a punto de bloquearse.

2. Barras de frenado (válvulas): Línea del líquido de frenos controlado por el ABS. Presuriza o libera presión en el neumático que lo requiera.

3. Unidad hidráulica (bomba): Recupera la presión que se liberó en los frenos mediante las válvulas.

4. Computadora ABS: Recibe las señal de los sensores de velocidad de las ruedas, con esta información opera las válvulas [4].

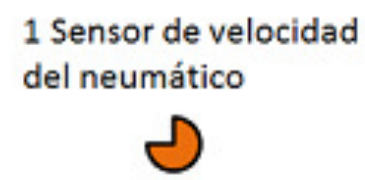

\section{Barras de frenado}

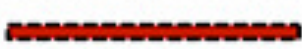

\section{Unidad hidráulica}

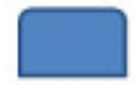

4 Computadora ABS
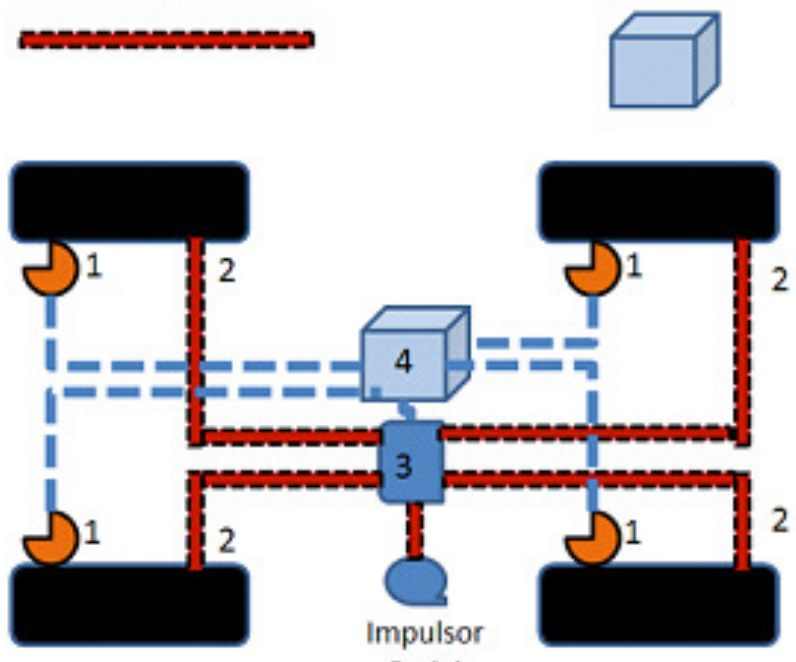

Pedal

Fig. 1. Diagrama principal de un ABS típico [3]. 
Una FPGA (Field Programmable Gate Array) es un dispositivo semiconductor que contiene campos de matrices de compuertas programables. Se caracterizan por altas densidades de compuertas, alto rendimiento y un número muy grande de entradas/salidas definidas por el usuario, tienen un esquema de interconexión flexible y su entorno de diseño similar a una matriz de puertas, no se limitada a la típica matriz AND-OR [5]. Además de contar con una matriz interna configurable de relojes lógicos. La FPGA tiene la ventaja de ser re-programable (lo que añade una enorme flexibilidad al flujo de diseño), su costo de desarrollo y adquisición son bajos y el tiempo de desarrollo es también menor. El proyecto consiste en tomar la señal de activación del ABS y enriquecerla con la señal de coeficiente de fricción adquirida en otro módulo, usando visión por computadora mismo que aquí no se detalla pero que se simula su señal, para que pueda ser activado el ABS tomando en cuenta el tipo de superficie, de esta manera estamos conjuntando a través de la FPGA el sistema de visión con el sistema ABS.

\section{Desarrollo}

En este proyecto se busca generar una señal enriquecida que active el sistema ABS de un automóvil incorporándole una señal de coeficiente de fricción, calculado a partir del procesamiento de imágenes usando lógica difusa, lo cual enriquece la señal para la activación del freno.

La figura 2 muestra la metodología del proyecto. En el inciso (A), puede observarse el comportamiento normal que sigue la activación del freno ABS. En el inciso (B), puede observarse cómo se integra la integración de la señal desde módulo de visión proveniente de la cámara, la cuál se ingresa al módulo de lógica difusa, dónde se determina el coeficiente de fricción, éste coeficiente de fricción será añadido a la señal proveniente del escáner del sistema de frenado ABS para enriquecerla [6], [7]. El coeficiente de fricción tiene dos posibles valores, 0 y 1. El 0 significa que el valor del coeficiente de fricción es mayor al límite (es decir, no hay deslizamiento) y por lo tanto, no se requiere activar el sistema ABS, en caso contrario su valor será de 1 .

Para el proyecto se han considerado tres posibles escenarios, los cuáles van acorde a los tipos de terrenos de desplazamiento del vehículo en la ciudad: (i) suelo seco y luz de día, (ii) suelo húmedo y luz de día, y (iii) suelo seco y luz baja.

Realizada la adquisición y análisis del video, el sistema propone el cálculo de un coeficiente de fricción asociado a la superficie de rodamiento. Este cálculo es estimado a partir del análisis de la propia imagen adquirida del video [8], y de esta forma se puede obtener una señal adicional, que complemente o coadyuve a la señal de la computadora del sistema ABS ante condiciones no supervisadas por el conductor del vehículo.

Las variables de entrada seleccionadas fueron la velocidad $(v)$, la entropía (e) y la luminosidad $(l)$; con éstas variables se configuró un sistema MISO (Multiple Input Single Output), es tableciendo los conjuntos difusos y reglas de 
(A)

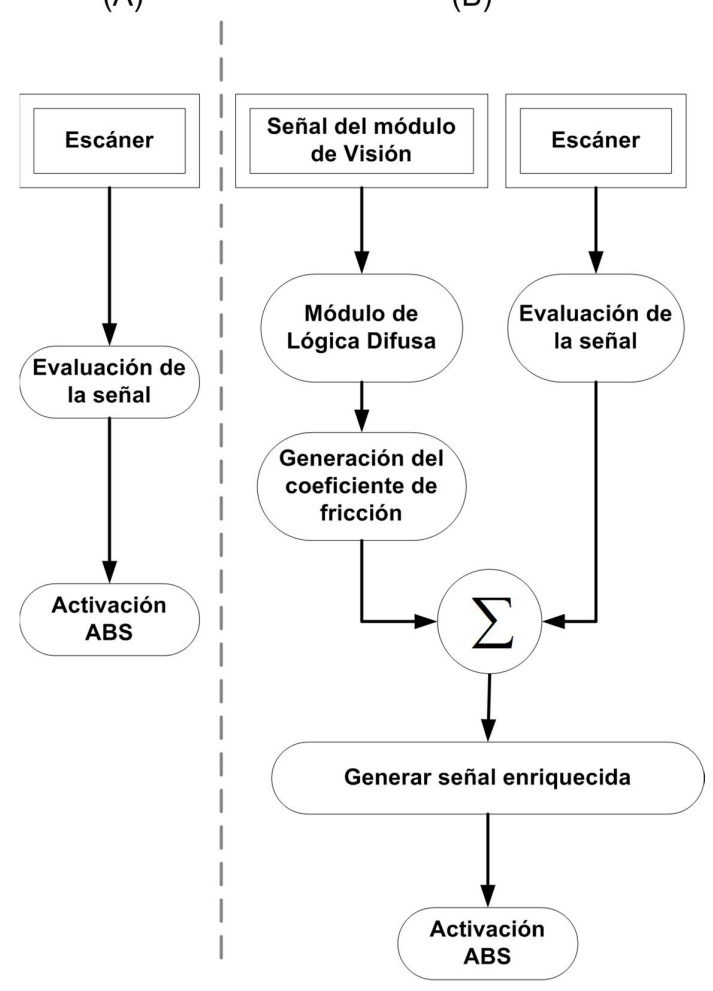

Fig. 2. Metodología del proyecto. (A) Funcionamiento de ABS. (B)Funcionamiento de ABS con señal Enriquecida. 
inferencia que permitieran su implementación real. Las características-variables consideradas $(v, e, I)$ fueron extraídas usando imágenes cuadro a cuadro del video obtenido por la cámara, para identificar las tres condiciones de superficie de rodamiento consideradas, $(i$, ii, iii). Del procesamiento de cada imagen se obtienen los valores de las tres variables $(v, e, I)$, que se ingresan al sistema difuso, y de acuerdo a un conjunto de reglas de inferencia se obtiene el coeficiente de fricción.

Combinando el coeficiente de fricción que da una señal con valor lógico de 0 ó 1 y las señales de activación procedentes del escáner, obtenemos una señal enriquecida. Esto se hará utilizando la siguiente tabla de verdad mostrada en la tabla de verdad mostrada en la figura 3 , donde se muestra el flujo de datos como señales que ingresan al dispositivo FTPS, se procesan y entregan como salida la activación o no del freno ABS.

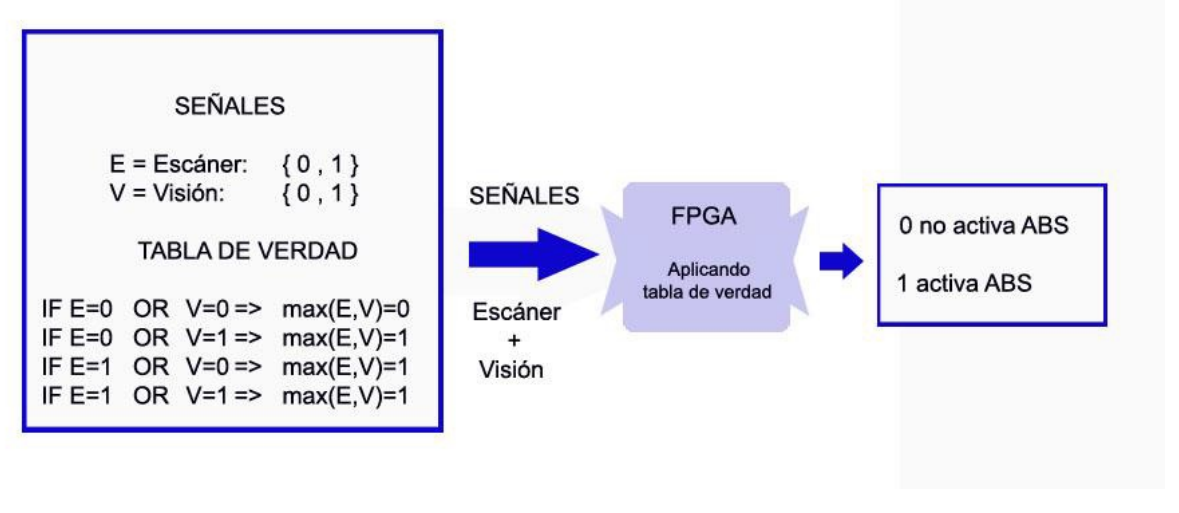

Fig. 3. Tabla de verdad de las señales de activación del ABS.

Se tienen dos variables de entrada, las imágenes de la cámara de video y la señal propia del sistema ABS. El objetivo del procesamiento de la señal de la cámara es ayudar al conductor en el dado caso de que no haya considerado correctamente la superficie sobre la cuál se encuentra y en caso de ser necesaria la activación del freno ABS, activarlo aunque el conductor no haya reaccionado a tiempo, con lo que se pretende evitar accidentes.

Otro aspecto tomado en cuenta es la velocidad de adquisición de señales. La lógica que se siguió es que a menor velocidad del vehículo la ayuda del sistema de activación ABS con señal enriquecida es menor. Por lo tanto, la velocidad de adquisición es directamente proporcional a la velocidad del vehículo. La tabla 1 muestra las velocidades de procesamiento establecidas.

Se considera que el tipo de superficie está asociado a un peso en las reglas de activación. En la Tabla 2, se aprecia el peso de defuzzificación asignado dependiendo el tipo de superficie/camino que se recorre. De igual manera, dentro 
José Vázquez Quezada, Juan Villegas-Cortez, Iván Vázquez-Álvarez, Carlos Avilés-Cruz, et al.

Tabla 1. Velocidad de procesamiento de la señal.

\begin{tabular}{|c|c|c|}
\hline $\begin{array}{ll}\text { Velocidad } & \text { longitu- } \\
\text { dinal del } & \text { vehículo } \\
(\mathrm{km} / \mathrm{h}) & \\
\end{array}$ & Valor del reloj & Frecuencia de procesamiento $(\mathrm{Hz})$ \\
\hline $1-19$ & 250 & 4 \\
\hline $20-49$ & 125 & 8 \\
\hline $50-79$ & 62 & 16 \\
\hline $80-119 \mathrm{Km}$. & 31 & 32 \\
\hline 120 o más & 16 & 62 \\
\hline
\end{tabular}

del procesamiento se incluye la eliminación de ruido por parte de la adquisición de imágenes por video, con la finalidad de eliminar los falsos positivos que pueden ser provocados por efectos de luz y sombras, para ello se evaluan 10 cuadros o imágenes consecutivas para evitar que una sombra o un reflejo puede ocasionar un 1. Esto significa que por cada segundo se obtienen tres coeficientes de fricción que son enviados a la señal de activación ABS. El tiempo que se toma para el muestreo de las señales es determinado dependiendo la velocidad del automóvil cada .015 segundos alrededor de 66 tomas por segundo, lo cual es suficiente para tomar las muestras necesarias para el cálculo del coeficiente de fricción.

Tabla 2. Tipos de superficie y pesos asignados.

\begin{tabular}{|c|c|}
\hline Tipo de superficie representada & Peso w(x) \\
\hline Superficie resbaladiza (aceite o hielo) & 0.8 \\
Superficie mojada & 0.5 \\
Superficie seca & 0.3 \\
\hline
\end{tabular}

Se realizaron pruebas de funcionamiento del sistema, se modificaron las variables de velocidad del automovil para verificar la asignación proporcional en la velocidad de procesamiento de la señal y la variable de activación de cámara mediante la captura de imágenes asociadas a diferentes tipos de suelo.

La simulación en el freno ABS fue realizada en laboratorios de Inteco Inc., controlado por Matlab, dicho sistema permite probar el algoritmo en un prototipo de una rueda. Este prototipo base el cual es usado para simular la llegada de las señales de activación del freno ABS. Se realizaron diversas pruebas aleatorias de de la señal del pedal y la señal que se recibirá de la cámara para activar el freno, obteniendo resultados satisfactorios ya que la activación manual coincidió con los obtenidos por el sistema, que se discutiran a continuación. 
Enriquecimiento de señal para activación de frenos ABS en automóviles

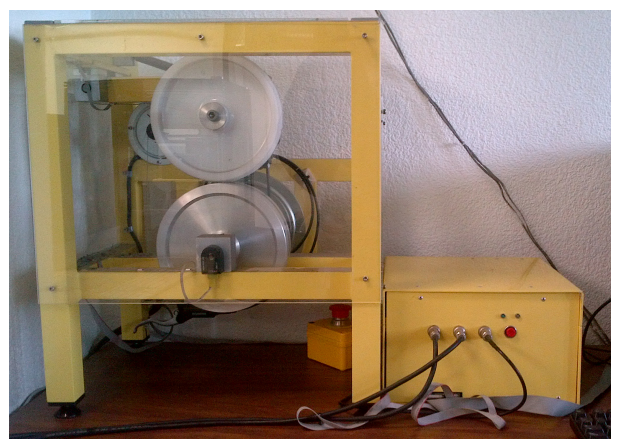

Fig. 4. Laboratorio ABS de Inteco Inc.

\section{Análisis de resultados}

En la figura 5, se muestra un ejemplo del estado de las señales para saber cuál debe ser el resultado esperado en la señal enriquecida, como se describe a continuación:

(a) Señal de freno en 0, señal de cámara en 0, señal enriquecida en 0.

(b) Señal de freno en 0, señal de cámara en 1, señal enriquecida en 1.

(c) Señal de freno en 1, señal de cámara en 0, señal enriquecida en 1.

(d) Señal de freno en 1, señal de cámara en 1, señal enriquecida en 1

Con el diagrama que se mostró anteriormente en la figura 2, en el DeviceDriver del Abs Main se obtiene la señal enriquecida que se estaba buscando. A continuación se muestran algunas de las gráficas que resumen el estándar de más de 100 corridas en ellas se puede ver una acotación promedio de los resultados obtenidos del procesamiento de estas dos señales. En la imagen se tiene primero la señal del pedal, segundo la señal de la cámara y tercero la señal enriquecida producida por las dos anteriores.

En la gráfica se aprecian las señales que activan el freno ABS cuando está en 1 y lo desactiva si estas en 0 , el eje de las X está delimitada la gráfica con el tiempo que transcurre en la simulación que es de (1000 milisegundos) y respecto al eje Y se tiene el valor de la señal enviada al freno desde la cámara, el pedal y la señal enriquecida que es de 0 ó 1 pero se toma en la gráfica de -.5 a 1.5 para poder apreciar de manera clara la señal.

Con esto se comprobó que la señal enriquecida se obtiene eficientemente. Como se muestra en la figura 5, de arriba hacia abajo se tiene la señal del pedal, señal de cámara y señal enriquecida.

La gráfica muestra la media de activaciones al simular una superficie con charcos o semi-mojada se puede ver como es más activa la señal de la cámara, también se realizó para una superficie casi perfecta, tomando como referencia una carretera sin baches, desniveles, sin basura o charcos en ella y para una superficie normal (tomando como normal una calle sin baches de nuestra ciudad). 


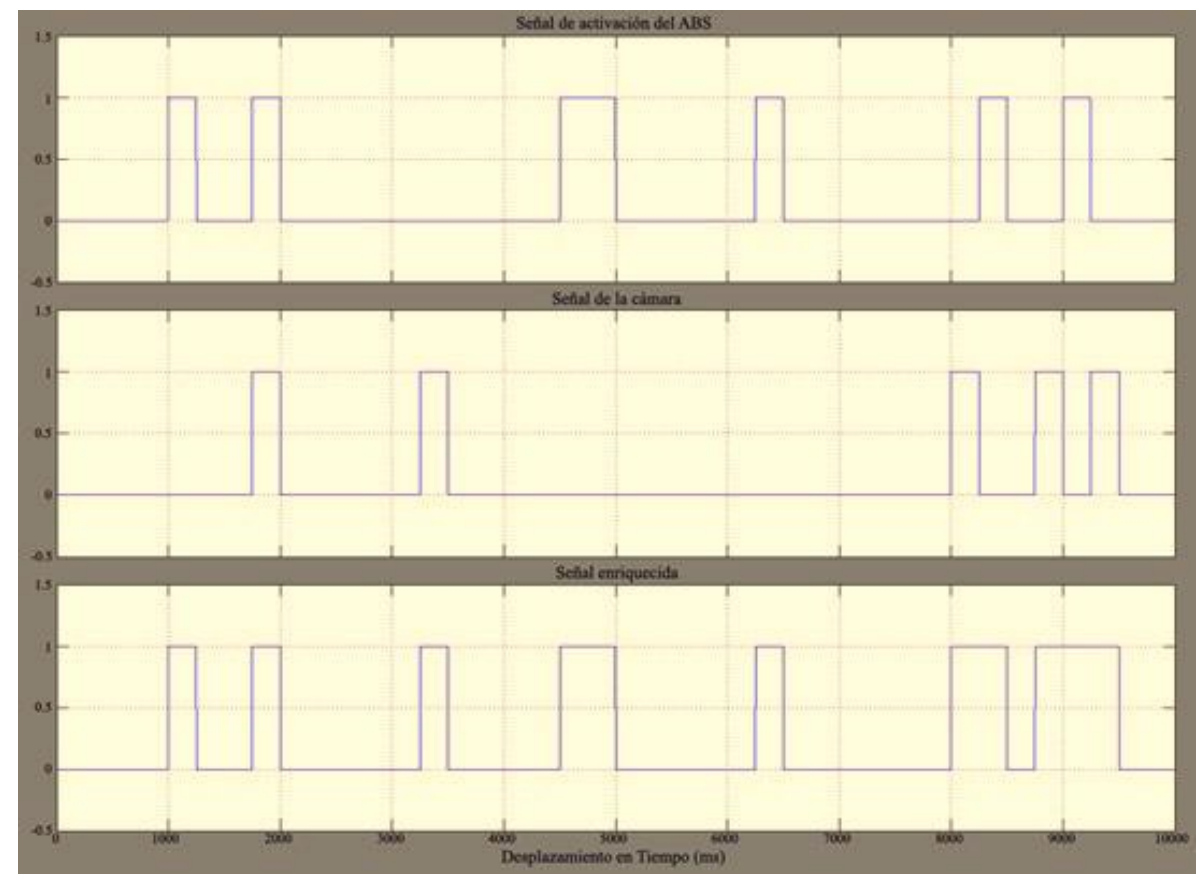

Fig. 5. Señal enriquecida de activación del ABS

Con lo que se puede afirmar que se obtuvo la señal enriquecida y su desempeño es factible y seguro de acuerdo a lo esperado para activar el freno del automóvil. Dejando una gran apertura a la implementación de muchas más señales que ayuden al control de seguridad del automóvil.

\section{Conclusiones y trabajo futuro}

En este trabajo se presentó una propuesta de mejora del desempeño del sistema ABS, mostrando cómo adicionar una señal generada por un sistema de visión por computadora externo, con la finalidad de ayudar a enriquecer de manera visual y mecánica la activación de éste; lo que se comprueba al integrar dicha información es la mejora del tiempo de respuesta de activación del freno ABS, ya que en lugar de esperar a que se inicie el proceso de frenado por parte del conductor y se presente una situación cercana al bloqueo, en su lugar se detecta una situación de riesgo, dadas las condiciones de la superficie en que se circula. Las condiciones de activación obtenidas por el sistema de visión han sido simuladas efectivamente, y con estas condiciones se ha validado el funcionamiento del sistema propuesto.

Un resultado obtenido es que puede prolongar la vida útil del freno reduciendo sus costos de mantenimiento, siendo un extra al aumento de su nivel de seguridad. 
También se puede hacer mención de la posibilidad de anexar más señales al sistema propuesto, y esto da pie a la incorporación de otras opciones diversas de señales para activar el freno, e.g. cámaras de visión traseras y laterales que se trabajan en sistemas para estacionar vehículos, o más sensores colocados en los neumáticos entre muchas otras. Acorde con los resultamos mostrados se obtuvo el desempeño esperado en la simulación con la instrumentación de laboratorio.

\section{Referencias}

1. Nieto, J.W.: Diseño y adaptación de un sistema de seguridad active para estacionamiento vehicular y monitoreo continuo. Technical report, Escuela Politecnica del Ejercito - Ecuador (2012)

2. Albert, M.P.: Frenos ABS. Alfaomega (1995)

3. BOSCH: Automotive Handbook. Bentley Publishers (2007)

4. Maosheng, Y.: Road surface condition detection and indentification and vehicle anti-skid control. Master's thesis, Cleveland State University (2008)

5. López, F.J.P.: Implementación de control por modos deslizantes para sistema abs. Proyecto termina división de ciencias básicas e ingeniería, Universidad Autónoma Metropolitana Unidad Azcapotzalco (2010)

6. Wang, J., Song, C., Jin, L.: Modeling and simulation of automotive four-channel hydraulic abs based on amesim and simulink/stateflow. In: Intelligent Systems and Applications (ISA), 2010 2nd International Workshop on. (2010) 1-4

7. Hernández, J.J.O.: Aplicación de control por modelo inverso a sistema abs. Proyecto terminal, división de ciencias básicas e ingeniería, Universidad Autónoma Metropolitana Unidad Azcapotzalco (2011)

8. Gonzalez, R.C., Woods, R.E.: Digital Image Processing. Addison-Wesley Longman Publishing Co., Inc., Boston, MA, USA (1992) 